\title{
Vehicle-to-Grid Technology: State-of-the-Art and Future
}

\section{Scenarios}

\author{
Alfonso Damiano, Gianluca Gatto, Ignazio Marongiu, Mario Porru and Alessandro Serpi \\ Department of Electrical and Electronic Engineering, University of Cagliari, Cagliari 09123, Italy
}

Received: July 20, 2013 / Accepted: September 09, 2013 / Published: January 31, 2014.

\begin{abstract}
An overview of V2G (vehicle-to-grid) technology is presented in this paper. It aims to highlight the main features, opportunities and requirements of V2G. Thus, after briefly resuming the most popular charging strategies for PEVs (plug-in electric vehicles), the V2G concept is introduced, especially highlighting its potentiality as a revenue opportunity for PEV owners; this is mainly due to the V2G ability to provide ancillary services, such as load leveling, regulation and reserve. Such solutions have been thoroughly investigated in the literature from both the economic and technical points of view and are here reported. In addition, V2G requirements such as mobility needs, charging stations availability and appropriate PEV aggregative architectures are properly taken into account. Finally, future developments and scenarios have also been reported.
\end{abstract}

Key words: V2G (vehicle-to-grid), EV (electric vehicles), BEV (battery electric vehicle), mobility, regulation.

\section{Introduction}

Thanks to the increasing environmental awareness and to the will to reduce both the dependence on fossil sources and the emission of greenhouse gases, the energy policy of many governments around the world is oriented to strongly support the expansion of RESs (renewable energy sources). In particular, the EU (European Union), through its Energy and Climate Policy, has set an ambitious target: to provide, by 2020, $20 \%$ of gross energy consumption from RESs. In particular, the EU has imposed, as a mandatory constraint, $10 \%$ of the overall energy consumption of the transport sector will have to come from RESs. This could be achieved by means of bio-fuel and EVs (electric vehicles). Although the first solution seems to be more rapidly viable, the second one provides many more opportunities from the technical, economic and social points of view.

EVs can be roughly divided into two big classes [1],

Corresponding author: Alfonso Damiano, associate professor, research fields: electrical machines, electric energy management and electric vehicles. E-mail: alfio@diee.unica.it. as shown in Fig. 1: PEVs (plug-in EVs) and non-plug-in EVs, which are essentially HEVs (hybrid EVs). In addition, PEVs can be further split into PHEVs (plug-in hybrid EVs) and BEVs (battery EVs). While BEVs are characterized by the use of batteries to supply their electric propulsion system, PHEVs are generally equipped with smaller batteries because they employ an internal combustion engine; this can directly propel the vehicle and/or recharge the battery on board, resulting in an extended mobility range compared to BEVs.

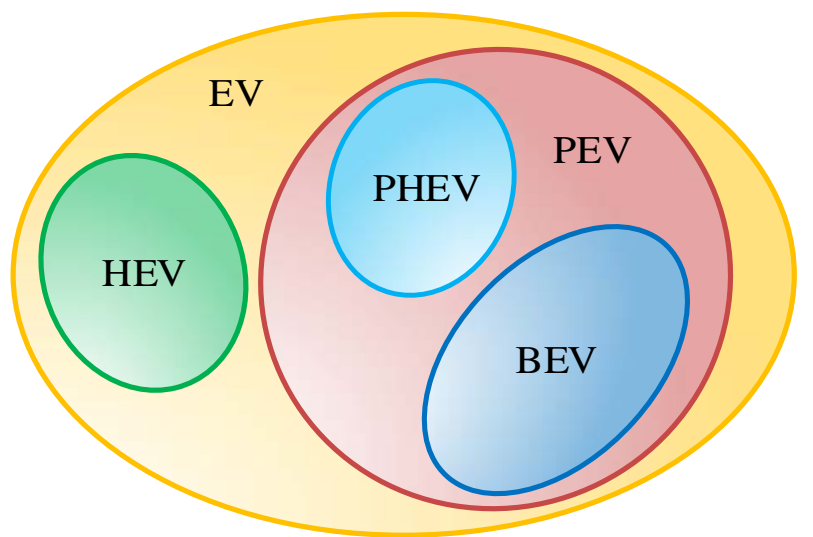

Fig. 1 Electric vehicles classification. 
At the present time, the most widespread EVs on the market are HEVs and, to a lesser extent, PHEVs. In fact, although BEVs present several advantages compared to ICEs (internal combustion engine vehicles), HEVs and PHEVs, such as favorable fuel economy, lower maintenance costs and reduced emissions [2-4], their use is not yet widespread; this is mainly due to the fact that their batteries are quite expensive and provide a low mobility range. This drawback could be overcome through the exploitation of the PEV batteries by means of appropriate charging strategies. These aim to minimize PEV operating costs and, hence, to partially compensate for the higher investment costs compared to ICEs and HEVs [2]. A viable and quite promising solution would appear to be V2G (vehicle-to-grid) technology [5-7]: This mainly consists of maximizing PEV owners' profits by providing several services to the electric grid $[2,8]$. This should allow many more benefits than by using optimal charging laws only.

In this paper, the most important V2G features, opportunities and requirements are summarized. In particular, the aim is to give an overview of the different solutions to exploit PEV batteries by means of V2G proposed in the literature, highlighting the pros and cons, from both the economic and technical points of view. The paper is structured as follows: the most popular PEVs charging strategies and the V2G basic concepts are briefly summarized in Section 2; then, V2G revenue opportunities are detailed in Section 3 and V2G requirements are reported in Section 4. In conclusion, V2G future developments are presented in Section 5 and final remarks are presented in Section 6.

\section{PEVs Battery Management}

Although the charging of a single PEV is not able to significantly affect the power system operation, a large number of PEVs will require appropriate charging management strategies due to the conspicuous power and energy flows [2, 9].

In recent years, many studies have been carried out to assert EVs impact on the electric grid. Some of them state that charging a limited amount of PEVs will not require significant grid re-arrangements and new power plants [3, 10-12]. In fact, it is estimated that the increase of electricity demand due to the introduction of 4 million PEVs in California, i.e., 25\% of the overall car fleet, can be fully satisfied by the actual power plants [9]. This is further confirmed by Ref. [12], which states that an increase of the total electricity demand by $5 \%-8 \%$ due to PHEVs in 2030 will not badly affect the electric grid. However, the effects of EV diffusion depend on the features of the grid they are fed by, together with their charging system. In fact, if the same above-mentioned PEV penetration level occurred in 13 US regions and all PEVs were recharged at about $5 \mathrm{pm}$, this would require the installation of 160 new power plants [9].

Even if the total impact of the extra load due to EV charging does not negatively impact the production side, the same might not be said for the grid side. In fact, due to the dispersed nature of such loads, in time and space, a number of problems can occur locally, such as overloads or power quality issues [13]. In addition, the PEVs diffusion process could badly affect the hourly power demand too, especially regarding the increase of peak electricity demand. However, this would occur if all EVs were recharged all together at $4 \mathrm{~kW}$, i.e., three times the average US domestic load. Otherwise, if the same charge is properly distributed over 8 night hours, the average load will be about $1 \mathrm{~kW}$, and no peak increase will occur [3]. All these considerations highlight the need for suitable PEV charging laws, which unavoidably have to take into account PEV owners' habits.

\subsection{PEVs Charging Strategies}

The charging strategies reported in the literature can be mainly categorized as follows [3, 14]:

- Dumb charging, which consists in fully recharging PEVs at rated power whenever they are plugged-in;

- Delayed charging, which entails a time delay 
between the PEV plug-in and the start of its recharging in order to minimize electricity costs;

- Smart charging, which consists in recharging PEVs on the basis of the needs of both the owners and the system operator.

If dumb charging is employed, PEV owners are totally free to plug-in and charge their vehicles whenever they want. The other charging strategies can be performed either manually by the user or automatically. In the first case, each PEV owner is able to set the way in which the vehicle is recharged. Such choice may be influenced by the system operator by means of appropriate incentive policies, such as dual tariff programs. In the second case, which mostly entails smart charging operation, an active management system with a hierarchical structure establishes the charging profile in order to maximize economic or technical benefits for both the electric grid and the owners [10]. Although all the above-mentioned charging strategies can presently be afforded by the system operator, in a high PEV penetration scenario, all PEVs will not be able to charge simultaneously and/or at their maximum rate. As a consequence, smart charging laws will be unavoidable [15]. In particular, they will be able not only to avoid negative impact on the electric grid, but also to guarantee its better performances, as shown in Fig. 2 [14].

Regarding this, in Ref. [14], authors investigate the maximum PEV penetration level achievable by means

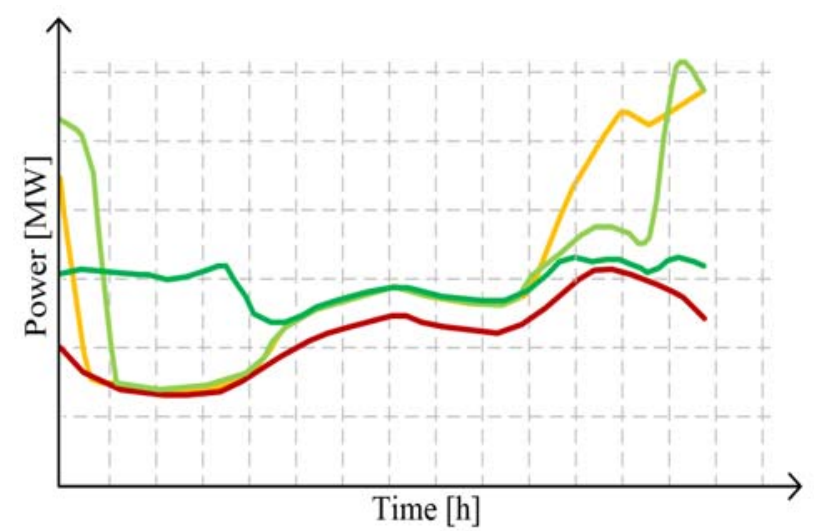

Fig. 2 Daily load profiles without EVs (red), with dumb charging (yellow), delayed charging (light green) or smart charging (dark green). of different charging strategies; in particular, grid parameters have been taken into account, such as node voltages, line capacities and losses. It has been shown that a smart charging strategy allows integration of a number of PEVs five times greater than that achievable by dumb charging. In addition, notwithstanding an increasing number of PEVs, nodes voltage and congestion levels do not increase due to the implementation of more sophisticated charging strategies. Moreover, losses can decrease and valleys can be filled at the same time [14].

\subsection{Vehicle to Grid Technology}

In order to enable their widespread use, PEVs have to guarantee mobility as close as possible to that provided by ICEs, entailing cost savings at the same time. A smart charge could meet this goal. In fact, due to the fact that PEV batteries charging period at rated power generally requires much less time than plug-in parking periods, it is possible to optimize the PEV charging process from the economical point of view [16]. However, PEV batteries can also be employed to reduce negative impacts of PEVs on power systems, even increasing their performances $[2,5,6,8,16]$. This can offer PEV owners the possibility to generate revenue by providing several services [8, 13, 14], while keeping their car plugged into the socket, which may lead to a rapid growth of PEV use. This is defined the V2G (vehicle-to-grid) concept [2, 11, 16].

There are two ways in which a PEV can operate V2G, depending on the power flow directions: Firstly when the power can flow from the grid to the PEV battery only, this operating mode is usually denoted by unidirectional V2G or G2V (vehicle-to-grid) [8, 10]. Secondly, when the power can be delivered from both sides, i.e., the grid and the PEV battery, such operating mode is called bidirectional V2G or, simply, V2G [5, 6, 10].

Although several services can be offered only by means of V2G, G2V can offer charging flexibility, load curtailment and, hence, frequency control [8 16, 17]. Thus, G2V gives PEV owners the opportunity to take part 
in the day-ahead market, buying energy and offering the services defined as "load only services". In addition, it is logical to suppose that $\mathrm{G} 2 \mathrm{~V}$ will be implemented earlier than V2G [5]. This is mainly due to the fact that it can be accomplished through standard J1772 chargers that are already available on the market, avoiding additional V2G hardware requirement, costs, performances and safety issues [17]. Moreover, G2V will not entail the overexploitation of PEV batteries due to uses other than PEV propulsion, whereas this issue will have to be taken into account by V2G. All these advantages result in lower implementation costs and faster returns on investment for G2V than for V2G $[8,17]$.

However, G2V can not exploit all the benefits that V2G can; in particular, due to the fact that G2V can provide services during recharging mode only, the ancillary services G2V can provide are quite limited. Such limitation generally leads to smaller profits (about 25\%) than those achievable by V2G [8, 17]. In fact, V2G allows PEV fleets to be considered as distributed ESSs (energy storage systems), the adoption of which is widely recognized as the most important improvement in the smart grid paradigm, increasing the grid's stability, flexibility and reliability $[5,6,17]$. As a consequence, PEVs will be able to address many issues, from RESs integration to micro-grid and islanded operating management.

\section{V2G Revenue Opportunities: Ancillary Services}

Ancillary services support the electricity transfer from the production to the loads with the aim of assuring power system reliability and enhancing power quality. However, although they are the same all over the world, there are no standard definitions; in fact, in some nations the same ancillary service has different names. The best-known ancillary services are regulation, voltage control, spinning and standing reserve $[18,19]$.

From the V2G point of view, ancillary services could be grouped into energy and power services, as shown in Fig. 3. In particular, energy services require significant amounts of energy to be delivered or absorbed by the service supplier. Hence, energy is purchased and sold back to the grid as needed. Power services entail a large power demand, with negligible energy exchanges. Consequently, the revenue comes from being able to deliver and/or absorb power over a certain period of time.

Therefore, due to the PEV characteristics, it would appear clear that V2G can compete mostly in the power services market $[2,5,6]$. In fact, PEVs are not particularly suitable for providing the significant amount of energy required by energy services. This is hard to manage by means of the small PEV battery rated capacities. More valuable services are regulation, i.e., frequency control, and reserve, which match V2G features, such as quick response, low specific power and stand-by costs well [5]. However, once these markets are saturated, V2G could provide energy services too, especially regarding load leveling, i.e., peak shaving \& valley filling, which are best performed by hydro pump power plants at present. Moreover, in future green power systems, which will be based on intermittent RESs, V2G could improve RES reliability and programmability, by enabling PEVs to operate as ESSs [2, 5, 6].

\subsection{Load Leveling}

Load leveling briefly consists in decreasing the peak electricity demand supplied by traditional power plants (peak shaving) and increasing the peak-off demand (valley filling) at the same time. This results in transferring a certain amount of electricity delivered by traditional power plants during a defined period of time, as shown in Fig. 4. This is valuable because flat load dispatching is easier than fluctuating load dispatching, simplifying forecasting and decreasing regulation needs [9].

It has been shown that V2G could contribute to both valley filling and peak shaving, but it is profitable only under certain circumstances [5]. In addition, load leveling requires careful planning based on matching 


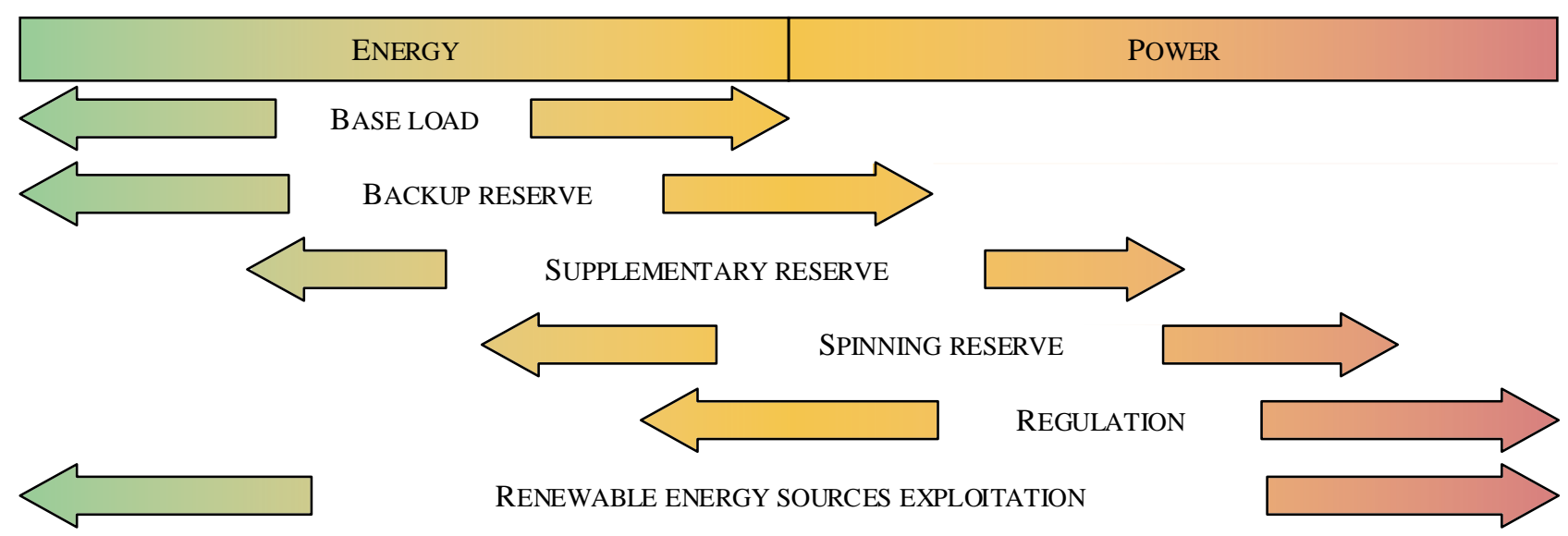

Fig. 3 Ancillary services classification.

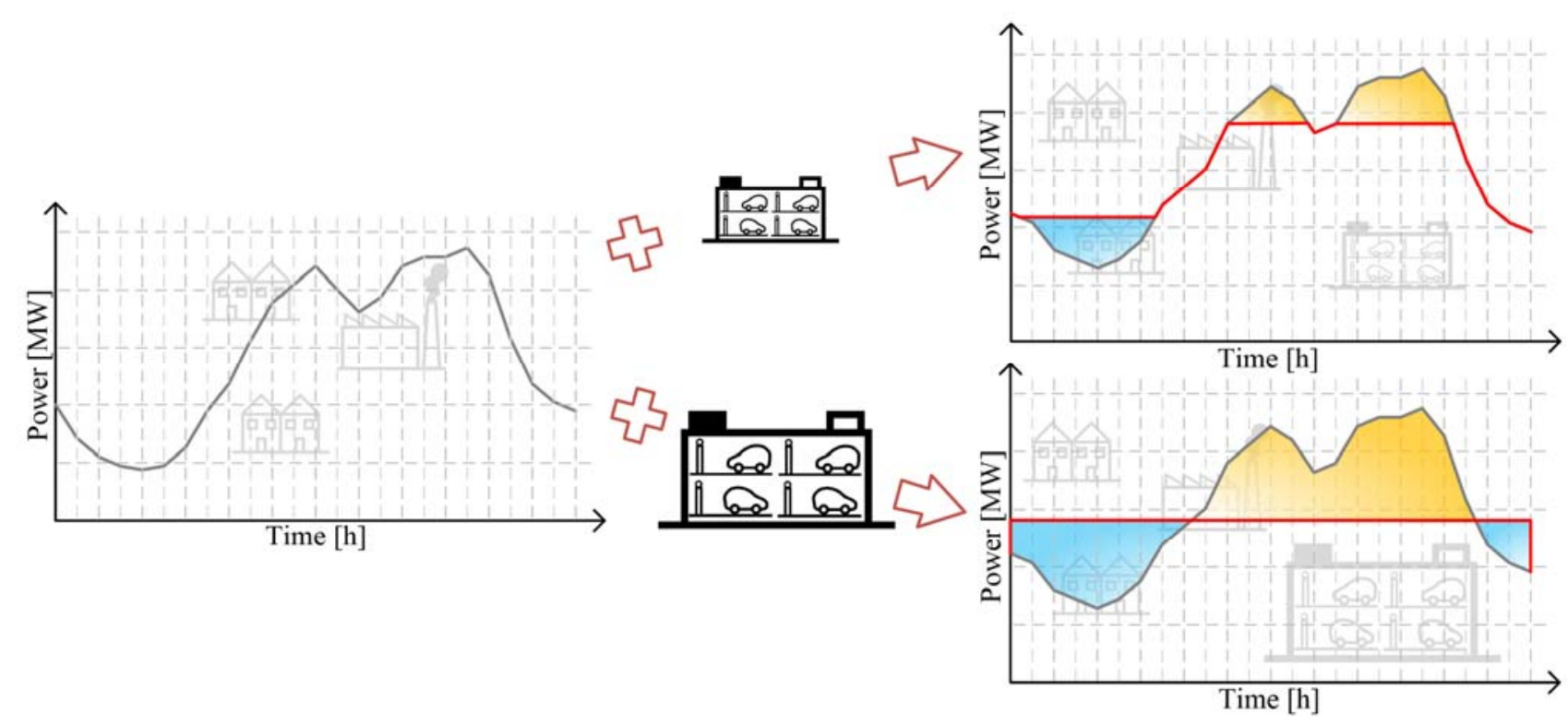

Fig. 4 Load leveling performance in case of small and large EV fleets providing V2G.

between PEV battery SOC and owners' mobility needs. On this point, a planning tool, suitable for finding the best compromise between mobility and V2G performances is proposed in Ref. [20]. In particular, referring to a given region and taking into account commuters' habits, the authors showed that $60 \%$ of the overall power is consumed in $11.5 \%$ of municipalities only. These are thus assumed ideal candidates for early V2G implementation, because PEVs will be able to shave the overall peak demand well, assuring a reasonable profit at the same time.

\subsection{Regulation}

Regulation or frequency control is the ancillary service used by the system operator to hold frequency within a given range. This is automatically accomplished in real time by varying some power plants' production level. In fact, they are forced by system operators to increase (regulation-up) or decrease (regulation-down) their production level [5, 18]. Regulation revenue consists of three parts: the capacity payment, which depends on the amount of the power bid; the service payment, on the basis of the overall exchanged energy; the opportunity cost payment, which has to account for the missing revenue due to reduced production level [2].

In order to enable V2G to provide regulation, several requirements would have to be satisfied, as 
stated in Ref. [10]. In particular, a proper framework that aggregates enough PEVs to reach the standard thresholds of minimum power, availability and reliability would be needed [10], allowing PEVs to access the market. This kind of framework would manage regulation bids on the basis of its PEV needs and potentialities [21]. In fact, it has been proved that higher regulation call rates and regulation services that entail no negligible energy exchanges can only just be provided by V2G. On this point, in Ref. [10], a PHEV electric range reduction of about $340 \mathrm{~km}$ per month has been estimated, leading to increased fuel consumption. This entails increased costs of about 11 per month, which represents $20 \%$ of the overall V2G benefits. However, several studies reported in the literature assume negligible energy exchanges, especially over the long-term. Moreover, it is supposed that PEVs exchange energy for just $10 \%$ of the overall service period, in accordance with current standards. Consequently, the case in which regulation can deplete PEV batteries is commonly neglected [5, $6,10,21]$.

Many studies state that V2G can generate profits in regulation market; in particular, in Ref. [5], it is shown that the total net profit for a single PEV (Toyota RAV4) could be 2,554\$, due to an annual revenue and costs of 4,928 and $2,347 \$$ respectively. A G2V approach is presented in Refs. [8, 16, 17]; in particular, regulation is provided by appropriately increasing or decreasing the PEV power connection level with respect to the POP (preferred operating point). As a result, in Ref. [16], it has been demonstrated that offering regulation with a daily demand of $20 \mathrm{kWh}$ allows EV owners to reduce by $15 \%$ the charging cost. However, regulation capability depends significantly on the choice of the POP: higher POP values entail higher regulation-down and lower regulation-up capability, and vice versa. In addition, it is worth noting that regulation-up corresponds to load curtailment and this can only be provided in accordance with PEV mobility needs. Furthermore, once fully recharged, PEVs can no longer provide regulation, until a journey occurs. Regarding this, in Refs. [8, 17], it has been shown that bidding regulation services by G2V may increase the peak demand: This is due to the fact that the POP has to be set to an average value, in order to successfully provide both regulation up and down, which are particularly required in such a period of the day. This drawback does not occur using V2G, since it is able to provide regulation even if PEVs are not charging.

Several studies have been presented in the literature that analyzes the performances achievable by V2G and G2V in providing regulation services $[2,16]$. The comparisons reveal different scenarios: in Ref. [16], V2G capability increases the revenue compared to G2V minimally, entailing much higher investments. Contrariwise, the economic analysis presented in Ref. [17] reveals that V2G can lead to profits which are extremely higher than those achievable by means of G2V. A very detailed study is reported in Ref. [2], in which both the V2G and G2V approach are considered for light-truck PHEV and BEV fleets. The study assesses potential costs and revenues for 250 vehicles with a daily trip of about $110 \mathrm{~km}$. Two different cases are considered, in which the fleets are able to provide regulation-down and both regulation-up and regulation-down by means of G2V and V2G, respectively. Furthermore, in order to make a fair comparison, the various solutions have been evaluated over a 10-year project. As a result, the combination of BEV and V2G solutions is the most economical: in fact, analyzing all the best cases, PHEVs can produce revenue of 1,250 \$ per vehicle per year (9.5\% of the total cost), versus $1,400 \$$ achievable by BEVs (10.7\%). On the other hand, G2V provides only $700 \$(5.4 \%)$ and $907 \$(7.0 \%)$ in the case of PHEV and BEV fleets, respectively. Furthermore, influences of rated power and capacity of PEV batteries on both V2G and G2V performances have been evaluated. It has been revealed that V2G revenues are more affected by power than by capacity, especially due to the high battery cost. 


\subsection{Reserve}

Although regulation and reserve would appear to be very similar ancillary services, they occur in different situations: in fact, regulation has to support the system in real time continuously, whereas reserve consists in an additional generating capacity that must be kept aside to cope with sudden power losses or load increases. Based on its response time, reserve services can usually be grouped into three classes, as shown in Fig. 5:

- spinning reserve, which has a response time from few seconds to 5-10 min;

- supplementary reserve, which has a response time from 5-10 min to half an hour;

- backup reserve, which cannot be quickly ready, but can operate over long periods of time.

The above-mentioned boundaries are not standardized, but they can change depending on the system operator. Generally, spinning reserve must be able to respond very quickly $[5,18]$, but it is not required to operate for long periods of time. In fact, spinning reserve is replaced by supplementary reserve and, in turn, by backup reserve as soon as they are available [18]. All reserve services are remunerated for power availability and for the energy delivered.

As for regulation, reserve can be profitable for V2G, mainly due to remunerated power availability. The possibility to provide spinning reserve, supplementary reserve or backup reserve depends on the energy availability of PEV fleets. In particular, a small number of PEVs is not be able to provide large amounts of energy, but can just contribute to spinning reserve markets.

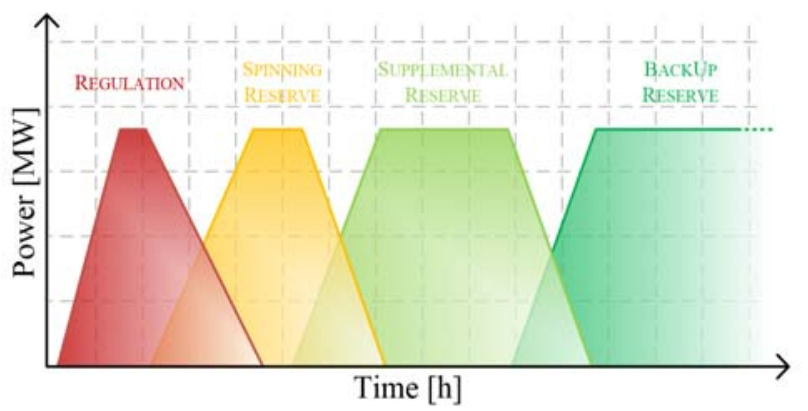

Fig. 5 Reserve classification.
G2V and V2G provide spinning reserve in very different ways. In fact, spinning reserve is provided with G2V by decreasing the power drawn from the scheduled POP, whereas V2G is able, not only to reduce its power drawn, but also to deliver energy from the PEV batteries.

\subsection{Renewable Energy Sources Exploitation}

The desire to depend less on fossil fuels, together with the need to reduce the emissions of greenhouse gases, requires the increase of electricity produced by RESs, especially by wind and photovoltaic power plants. The latter have quite a regular daily cycle, their production peaks occurring about $4 \mathrm{~h}$ before the load peak. On the other hand, wind power plant production is much less predictable, strongly sensitive to geographical location too. At the present time, there is a strong penetration of wind power plants and it is expected that it will further increase in the coming decades. However, both these kind of RESs are characterized by poorly predictable energy production profiles, together with highly variable rates. As a consequence, the electric grid can not manage these intermittent power sources beyond certain limits, resulting in RES generation curtailments and, hence, in a RES penetration level lower than is expected.

Several solutions that aim to increase RES integration have been proposed in the literature, which resort to deferrable loads [22], appropriate ESSs [23-27] or V2G technology [6, 23, 28, 29]. This last solution appears to be very promising, as shown in Fig. 6. In fact, ESSs fully devoted to RES exploitation may be quite expensive, so one of the most important advantages of V2G is that batteries will be already available because they are purchased for another purpose, i.e., the PEVs mobility. However, dealing with not-devoted ESSs requires appropriate management systems in order to successfully predict their SOC and availability [30].

An early estimation of the number of PEVs for RES integration is reported in Ref. [6]. In particular, two 

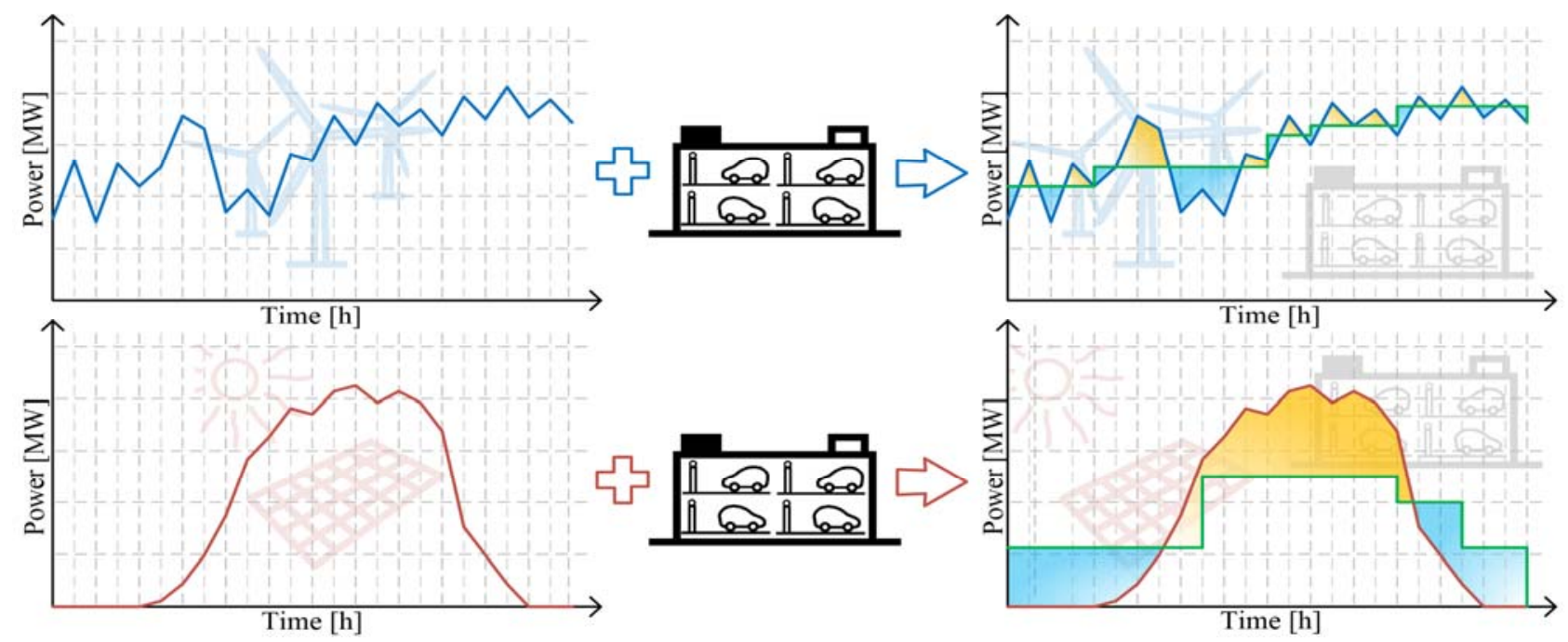

Fig. 6 Renewable energy sources exploitation by V2G.

different methods are considered; the first one consists in determining the size of the PEV fleet in order to fully integrate photovoltaic power plants only by means of peak shaving. It is shown that 143 PEVs (Toyota RAV4, $27.4 \mathrm{kWh}, 15 \mathrm{~kW}$ ) are needed to integrate 1 MW photovoltaic power plant. Extending this result, if one fifth of $811 \mathrm{GW}$ power plants in the US is converted into photovoltaic power plants, it will require $164 \mathrm{GW}$ of V2G power, which corresponds to $13 \%$ of the national car fleet [6]. The second method proposed in Ref. [6], which concerns wind power plants only, is based on the assumption that increasing electric power that comes from wind power plants increases regulation needs. In particular, it is estimated that regulation needs to grow by $6 \%$ and by $11 \%$ in order to integrate large and small wind power plants, respectively [6]. Hence, assuming half of the whole US energy production is coming from wind power plants (700 GW), a lower regulation boundary of $6 \%$ is imposed (42 GW). This can be provided by V2G resorting to a PEV fleet which will be just $1.6 \%$ of the overall US car fleet.

A V2G approach with the aim of maximizing RESs exploitation is also proposed by Ref. [31]. In particular, the study refers to a weakly interconnected power grid, characterized by a strong RESs penetration level. The management strategy aims to store as much as possible the RES production which exceeds that which is allowed by the system operator. Then, such stored energy is employed to propel PEVs and/or provide ancillary services (reserve, backup power). In particular, it has been estimated that 10,000 PEVs (1\% of the overall car fleet) could increase RESs exploitation to $98.8 \%$ with just $4 \mathrm{~kW}$ rated power, basically due to reserve services [1].

An algorithm to maximize RES usage in charging PEVs is proposed in Refs. [4, 30]. It consists in firstly determining optimal charging periods in order to recharge PEVs by means of wind power plants as much as possible, taking into account PEV owners' habits and needs (wind-to-vehicle, W2V). Subsequently, a classical V2G approach is suggested in order to give back to the grid a share of the energy previously absorbed (wind-to-vehicle-to-grid, W2V2G). As a result, it has been shown that wind energy absorbed by PEVs can be increased from $12.8 \%$ to $20.7 \%$ and $22.7 \%$ by means of $\mathrm{W} 2 \mathrm{~V}$ and $\mathrm{W} 2 \mathrm{~V} 2 \mathrm{G}$, respectively. This also results in further reducing $\mathrm{CO}_{2}$ emissions, which decrease from $23 \%$ to $13 \%$ by means of W2V2G compared to those produced by ICEs [4].

\section{V2G Requirements}

It has been shown in the previous section that PEV owners can make a profit by V2G. This will be 
accomplished by satisfying several requirements, among which the most important ones are that V2G operation should not impair PEVs mobility and should benefit from appropriate charging infrastructures and control \& communication devices. And, last but not at least, suitable V2G architectures should be developed in order to aggregate a certain amount of PEVs. This is mandatory in order to provide some of the services previously mentioned.

\subsection{Mobility Needs}

One of the major drawbacks to managing PEV batteries by V2G is the random nature of owners' habits and the enslavement of PEVs to the needs of the power system rather than to those of their owners. However, even if a single PEV or a small number of them can be characterized by poorly predictable habits, these become less random as the size of the PEV fleet increases. In addition, it is worth pointing out that PEV owners can choose the V2G operation that best fits their needs, without having to change their habits.

In order to further corroborate the groundlessness of such fears, several studies have been carried out on the basis of ICE owners' habits. In fact, since PEVs are not yet widespread, there are not enough data for detailed analysis. However, vehicles electrification is not going to change people's lifestyles and habits, so ICEs data could be appropriately employed [13]. The most important information concerns:

- the number of cars on the road;

- when and where a car is parked;

- the distance traveled.

In a study of the UK Department of Transport (UK Time Use Survey), it is reported that people mainly use their car for the home-work-home route on week days, resulting in being on the road from 7-9 am and 4-7 pm. However, even during rush hours, no more than $11 \%$ of the whole fleet is on the road. It means that at least $89 \%$ of cars are parked [32, 33]. Moreover, the study estimates that the average distance covered by private cars during a travel is about $14.5 \mathrm{~km}$. It means that a mean trip would use approximately $10 \%$ of the SOC of a PEV characterized by $130 \mathrm{~km}$ range [33].

In spite of some differences, these results are corroborated by another study regarding the US transport system, that states that $85 \%-90 \%$ of vehicles are parked at home, work or shopping malls, being idle for $22 \mathrm{~h}$ per day [3, 9]. Furthermore, the same study estimates that an average roundtrip commuting distance of about $50 \mathrm{~km}$ is covered in about $52 \mathrm{~min}$. In addition, $80 \%$ of commuters travel less than $60 \mathrm{~km}$ per day [9].

A mobility modeling procedure suitable for V2G is proposed in Ref. [31]. A generic PEV fleet is split into three sub-fleets: The first is made up of all the PEVs on the road, the second consists of all PEVs parked and plugged in, whereas the last one groups all PEVs that are parked and unplugged. Obviously, individual PEV may move from one fleet to another, but not all the transitions are plausible, as illustrated in Fig. 7. It is worth noting that the number of PEVs belonging to the first sub-fleet depends on PEV owners' habits only, whereas those of the second and third sub-fleet are strongly affected by charging infrastructures too. The knowledge of PEV distribution among these sub-fleets is fundamental in order to provide V2G as well as possible, without impairing PEV mobility needs.

\subsection{Charging Stations}

PEV batteries exploitation by V2G will strongly depend on the amount and distribution of charging stations. In fact, appropriate planning of the charging stations distribution will increase PEV availability for V2G purposes, making V2G more flexible and reliable at the same time, overcoming the PEVs "range anxiety"

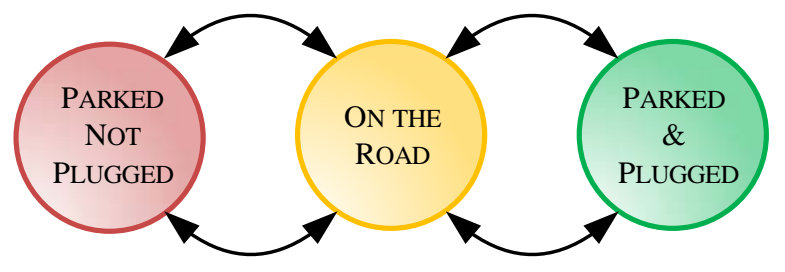

Fig. 7 The PEV sub-fleets classification. 
issues. Confirming this, in Ref. [11] PEV availability has been estimated with reference to the case in which each PEV owner provides V2G on his own, firstly from home only and, subsequently, from both home and work: this results in increasing PEV availability from $83.6 \%$ to $91.7 \%$ in the long-term.

In Ref. [3], it has been proved that wide distribution of charging stations, e.g., home, work and shopping centers, will bring more benefits than high power recharge rates; in fact, the domestic electricity demand decreases by about $5 \%-9 \%$ compared to the case in which charging is possible at both home and work, even by $24 \%-29 \%$ compared to the case in which charging is allowed at home only [3]. Furthermore, wide distribution of charging stations will extend the PEV charging process throughout the day, reducing the peak load by $20 \%-35 \%$ [3].

In conclusion, distribution of charging stations is particularly important for PHEVs: in fact, this constrained PHEV electric mode operation more than charging time and power rate do [3]. On the other hand, a wide distribution of charging stations will require a more accurate power system modeling and high investments [3].

\subsection{V2G Architectures}

Two main V2G architectures have been proposed in Ref. [11]. The deterministic architecture shown in Fig. 8, in which V2G is provided by each PEV autonomously, directly controlled and linked to the system operator by communication and power lines. The second one (aggregative architecture) consists of a framework providing V2G by means of a PEV fleet, as shown in Fig. 9. The first solution would appear to be simple and easy to implement, but it prevents V2G from providing several services that require high power and energy minimum thresholds. Contrariwise, an aggregative framework does not prevent V2G from delivering any services, but introduces additional costs.

Regarding profits, availability and reliability, both solutions have been considered in order to select, from

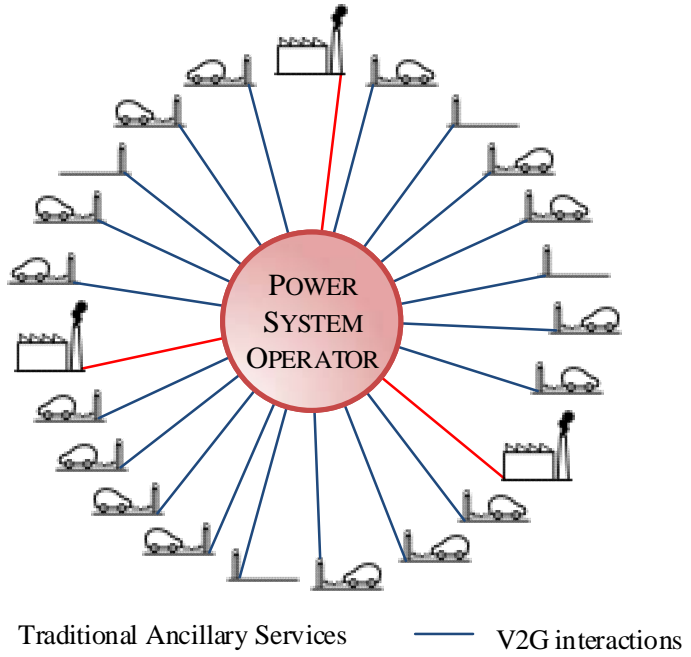

Fig. 8 A V2G deterministic architecture [11].

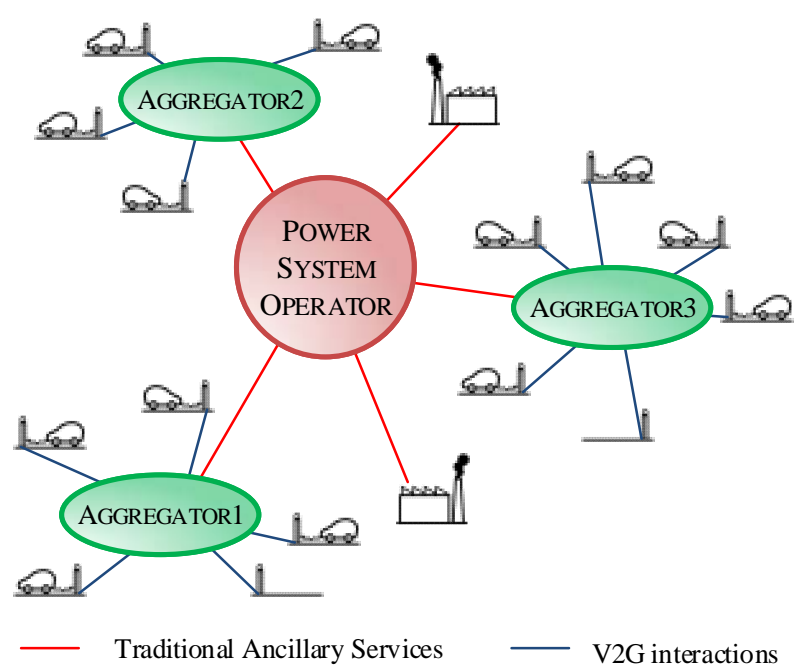

Fig. 9 A V2G aggregative architecture [11].

time to time, the most suitable one. In Ref. [11], the availability and reliability of these V2G architectures in providing ancillary services is estimated. It has been proved that the availability and reliability achievable by means of the deterministic V2G architecture is about $92 \%$ and $95 \%$ in the best cases, respectively. Whereas, they can both be assumed equal to $100 \%$ for the aggregative architecture, due to its inherent nature, which allows it to modulate the bidding on the basis of the state of the PEV fleet. Therefore, since the availability and reliability of base load generators is about $93 \%$ and $98.9 \%$ respectively, it can be stated that an aggregative architecture will be needed to meet industrial standards [11]. 
An aggregation of PEVs will be needed in order to participate in the energy market [9]. In fact, two different approaches can be followed: cost function-based power drawn scheduling and price-sensitive energy bidding [16]. The first one, which is suitable for the deterministic V2G architecture, consists in establishing the PEV charging profile on the basis of the energy price given by the day-ahead market and in updating it dynamically. As a result, each PEV is responsible for its charging without interference from the system operator: in the hours of cheapest prices, the PEV should recharge at its maximum rate. On the other hand, the price-sensitive energy bidding approach entails that the PEV fleet participates in the day-ahead market and the amount of energy purchased depends on the price the PEV owner is willing to pay [16]. This approach, which is not possible for the V2G deterministic architecture, is particularly suitable for the aggregative one. However, in both cases, PEVs can participate in the services markets.

Since the aggregative V2G architecture would appear to be the most promising one, several studies have been carried out aiming to define the role and tasks of this framework, which is also defined aggregator. Its role may be acting as an intermediary between each PEV owner and the system operator, whereas its tasks may consist in grouping a certain number of PEVs, appropriately coordinating their charging, and providing profitable services $[4,6,9,16$, 17].

The aggregator will be able to purchase energy more cheaply than a single PEV owner, thus its profits will consist of a pre-set share of all the revenue coming from the energy sold and the V2G services provided.

\section{Future Scenarios}

\section{$5.1 \mathrm{EVs}$}

The main features of future EVs can not be precisely stated as they will depend on many technical, economic, environmental and social issues. However, it appears plausible that many EVs models will be available to match different owners' needs and electric grid requirements [13]. For example, it is expected that the cost of batteries will decrease by $50 \%$ by 2020 , mainly due to large scale production and technological innovations. This would allow EVs to reach a 50\% market share by 2020, present market share being less than $10 \%$ [2]. In addition, improvements in battery technology will enable EVs to extend their range. On this point, it is expected that BEVs will have a range of $160 \mathrm{~km}$ at least [1]. Furthermore, the PEVs batteries capacity and power will be up to $70 \mathrm{kWh}$ and $20 \mathrm{~kW}$ respectively, and batteries will be fully recharged in 8-12 h or less [7, 9].

Assuming PEVs as one of the main elements of future power systems, four steps of evolution can be considered, as shown in Fig. 10. The first one concerns the EVs available today; being in their infancy, they can not be as mature as ICEs, but they can give important feedbacks to manufacturers to direct further and future research. Almost all of the current EVs allow unidirectional power flow and manual programmable charging operation. Communication capabilities are still very modest, since they only allow controlling EVs status from remote. In the US vehicle market, all manufacturers agreed to equip their vehicles with a common plug SAE J1772, available at level-1 (120 V) or level-2 (240 V) [1]. The second generation of EVs will be essentially based on the first one, but battery control and efficiency will be enhanced in order to increase their range and decrease their costs. In addition, even if power capability will remain the same, communication capability will be improved in order to implement more sophisticated charging strategies. Consequently, second generation EVs will be able to provide ancillary services by G2V mostly [1]. Considerably different from the first two generations will be the third one, since EVs will be characterized by high rated power and a bidirectional power flow charger. It is expected that charging will be performed at 50-100 kW, significantly reducing the recharge time. The bidirectional energy flow will allow EVs to operate 


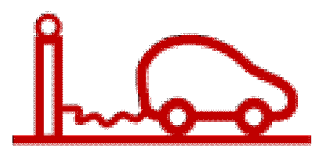

(a)


(b)
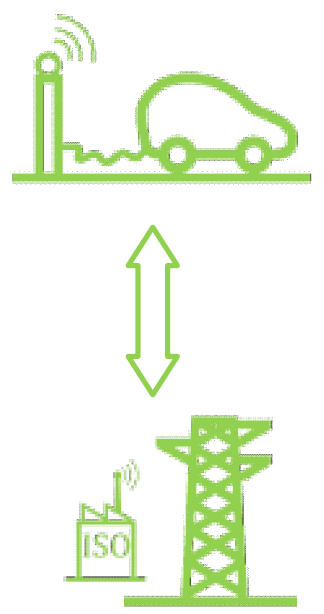

(c)
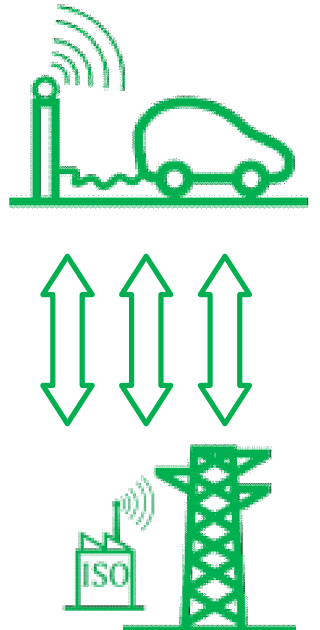

(d)

Fig. 10 Four steps of evolution for EVs.

V2G at a rudimentary level [1]. Finally, EVs of the fourth generation will have a significant V2G capability. They will also be equipped with an advanced and reliable communication system, guaranteeing their aggregation [9].

\section{$5.2 V 2 G$}

It has been shown in previous sections that the revenue achievable by V2G will be mainly determined by providing ancillary services; however, it is reasonable to expect that the profits will decrease as the number of PEVs providing such kind of services increase, leading to market saturations. In this regard, a comparative study on the Texas power system is reported in Ref. [17]. In this study, the effects of both G2V and V2G on ancillary service markets are considered. Based on G2V bidding capacity, a drop of $70 \%$ of the ancillary services market price has been estimated for just 300.000 PEVs, i.e., a share of only $1.5 \%$ of the 20 million registered vehicles in Texas. The same price reduction is achieved with just 100.000 PEVs providing V2G services. As a result, it is clear that market saturation could be reached very quickly [17]. This may well not be a significant drawback for $\mathrm{G} 2 \mathrm{~V}$, since its initial investment cost is quite low. In the case of V2G, however, market saturation could be achieved even before the payback time period.
Although the study refers to the regulation market only, it shows how the implementation of V2G requires careful economic evaluation, maybe thinking of new services that V2G should provide.

\section{Conclusions}

A wide use of PEVs will bring many benefits, but it may cause several drawbacks too. Choosing appropriate PEV management strategies will be needed in order to minimize PEVs operating costs and their impact on the power system. V2G would appear to be one of the most promising solutions. In particular, V2G is especially profitable in providing ancillary services, such as load leveling, regulation and reserve. Moreover, RESs support can be a viable alternative once the other services are saturated. This analysis of the literature reveals that different ways to perform V2G exist. Hence, the most suitable one should be carefully chosen on the basis of a holistic analysis, which depends on specific goals and local environments and takes into account technical, economic, planning and mobility aspects.

\section{Acknowledgments}

Mario Porru gratefully acknowledges Sardinia Regional Government for the financial support of her Ph.D. scholarship (P.O.R. Sardegna F.S.E. Operational 
Programme of the Autonomous Region of Sardinia, European Social Fund 2007-2013-Axis IV Human Resources, Objective 1.3, Line of Activity 1.3.1).

\section{References}

[1] D.P. Tuttle, R. Baldick, The evolution of plug-in electric vehicle-grid interactions, IEEE Trans. Smart Grid 3 (1) (2012) 500-505.

[2] A. De Los Rios, J. Goentzel, K.E. Nordstrom, C.W. Siegert, Economic analysis of vehicle-to-grid (V2G)-enabled fleets participating in the regulation service market, in: Proc. IEEE Innovative Smart Grid Technologies Conference (ISGT 2012), Washington D.C., USA, Jan. 16-18, 2012, pp. 1-8.

[3] C. Weiller, Plug-in hybrid electric vehicle impacts on hourly electricity demand in the united states, Energy Policy 39 (6) (2011) 3766-3778.

[4] J. Keiser, J. Glass, N. Masuch, M. Lutzenberger, S. Albayrak, A distributed multi-operator W2V2G management approach, in: Proc. IEEE International Conference on Smart Grid Communications (SmartGridComm), Brussels, Belgium, Oct. 17-20, 2011, pp. 273-278.

[5] W. Kempton, J. Tomic, Vehicle-to-grid power fundamentals: Calculating capacity and net revenue, Journal of Power Sources 144 (1) (2005) 268-279.

[6] W. Kempton, J. Tomic, Vehicle-to-grid power implementation: From stabilizing the grid to supporting large-scale renewable energy, Journal of Power Sources 144 (1) (2005) 280-294.

[7] L.J. Beck, V2G-101: A Text about Vehicle-to-Grid, the Technology which Enables a Future of Clean and Efficient Electric-powered Transportation, 2009.

[8] E. Sortomme, M.A. El-Sharkawi, Optimal charging strategies for unidirectional vehicle-to-grid, IEEE Trans. Smart Grid 2 (1) (2011) 131-138.

[9] C. Guille, G. Gross, A conceptual framework for the vehicle-to-grid (V2G) implementation, Energy Policy 37 (11) (2009) 4379-4390.

[10] C. Quinn, D. Zimmerle, T.H. Bradley, An evaluation of state-of-charge limitations and actuation signal energy content on plug-in hybrid electric vehicle, vehicle-to-grid reliability, and economics, IEEE Trans. Smart Grid 3 (1) (2012) 483-491.

[11] C. Quinn, D. Zimmerle, T.H. Bradley, The effect of communication architecture on the availability, reliability, and economics of plug-in hybrid electric vehicle-to-grid ancillary services, Journal of Power Sources 195 (5) (2010) 1500-1509.

[12] Environmental Assessment of Plug-in Hybrid Electric Vehicles, Volume 1: Nationwide Greenhouse Gas
Emissions, Tech. rep., EPRI, July 2007.

[13] S. Shahidinejad, S. Filizadeh, E. Bibeau, Profile of charging load on the grid due to plug-in vehicles, IEEE Trans. Smart Grid 3 (1) (2012) 135-141.

[14] J.A.P. Lopes, F.J. Soares, P.M.R. Almeida, Integration of electric vehicles in the electric power system, Proc. IEEE 99 (1) (2011) 168-183.

[15] Y. Hermans, B. Le Cun, A. Bui, Individual decisions \& schedule planner in a vehicle-to-grid context, in: Proc. IEEE International Electric Vehicle Conference (IEVC 2012), Greenville, USA, Mar. 4-8, 2012, pp. 1-6.

[16] M.A. Fasugba, P.T. Krein, Cost benefits and vehicle-to-grid regulation services of unidirectional charging of electric vehicles, in: Proc. IEEE Energy Conversion Congress and Exposition (ECCE 2011), Phoenix, USA, Sept. 17-22, 2011, pp. 827-834.

[17] E. Sortomme, M.A. El-Sharkawi, Optimal combined bidding of vehicle-to-grid ancillary services, IEEE Trans. Smart Grid 3 (1) (2012) 70-79.

[18] I. Kuzle, D. Bosnjak, S. Tesnjak, An overview of ancillary services in an open market environment, in: Proc. IEEE Mediterranean Conference on Control Automation, Athens, Greece, June 27-29, 2007, pp. 1-6.

[19] A.M. Pirbazari, Ancillary services definitions, markets and practices in the world, in: Proc. IEEE Transmission and Distribution Conference and Exposition: Latin America (TD-LA), Sao Paulo, Brazil, Nov. 8-10, 2010, pp. 32-36.

[20] A. Damiano, G. Gatto, I. Marongiu, A. Serpi, A vehicle to grid planning tool for weakly interconnected power systems, in: Proceedings of 10th International Conference on Environment and Electrical Engineering (EEEIC 2011), Rome, Italy, May 8-10, 2011, pp. 1-4.

[21] S. Han, S. Han, K. Sezaki, Development of an optimal vehicle-to-grid aggregator for frequency regulation, IEEE Trans. Smart Grid 1 (1) (2010) 65-72.

[22] A. Papavasiliou, S.S. Oren, Supplying renewable energy to deferrable loads: Algorithms and economic analysis, in: Proc. IEEE Power and Energy Society General Meeting, Minneapolis, USA, July 25-29, 2010, pp. 1-8.

[23] B.V. Mathiesen, H. Lund, Comparative analyses of seven technologies to facilitate the integration of fluctuating renewable energy sources, IET Renewable Power Generation 3 (2) (2009) 190-204.

[24] M.H. Nehrir, C. Wang, K. Strunz, H. Aki, R. Ramakumar, J. Bing, et al., A review of hybrid renewable/alternative energy systems for electric power generation: Configurations, control, and applications, IEEE Trans. Sustain. Energy 2 (4) (2011) 392-403.

[25] M. Swierczynski, R. Teodorescu, C.N. Rasmussen, P. Rodriguez, H. Vikelgaard, Overview of the energy storage systems for wind power integration enhancement, in: Proc. 
IEEE International Symposium on Industrial Electronics (ISIE 2010), Bari, Italy, July 4-7, 2010, pp. 3749-3756.

[26] T.K.A. Brekken, A. Yokochi, A. von Jouanne, Z.Z. Yen, H.M. Hapke, D.A. Halamay, Optimal energy storage sizing and control for wind power applications, IEEE Trans. Sustain. Energy 2 (1) (2011) 69-77.

[27] K. Heussen, S. Koch, A. Ulbig, G. Andersson, Unified system-level modeling of intermittent renewable energy sources and energy storage for power system operation, IEEE Systems Journal 6 (1) (2012) 140-151.

[28] Y. Ota, H. Taniguchi, T. Nakajima, K.M. Liyanage, J. Baba, A. Yokoyama, Autonomous distributed V2G (vehicle-to-grid) satisfying scheduled charging, IEEE Trans. Smart Grid 3 (1) (2012) 559-564.

[29] J.A.P. Lopes, P.M. Rocha Almeida, F.J. Soares, Using vehicle-to-grid to maximize the integration of intermittent renewable energy resources in islanded electric grids, in: Proc. IEEE International Conference on Clean Electrical Power (ICCEP 2009), Capri, Italy, June 9-11, 2009, pp.
290-295.

[30] N. Masuch, J. Keiser, M. Lutzenberger, S. Albayrak, Wind power-aware vehicle-to-grid algorithms for sustainable EV energy management systems, in: Proc. IEEE International Electric Vehicle Conference (IEVC 2012), Greenville, USA, Mar. 4-8, 2012, pp. 1-7.

[31] A. Damiano, I. Marongiu, M. Porru, A. Serpi, Electric vehicle energy storage management for renewable energy sources exploitation, in: Proc. IEEE International Electric Vehicle Conference (IEVC 2012), Greenville, USA, Mar. 4-8, 2012, pp. 1-8.

[32] F. Berthold, B. Blunier, D. Bouquain, S. Williamson, A. Miraoui, PHEV control strategy including vehicle to home (V2H) and home to vehicle (H2V) functionalities, in: Proc. IEEE Vehicle Power and Propulsion Conference (VPPC 2011), Chicago, USA, Sept. 6-9, 2011, pp. 1-6.

[33] Y. Ma, T. Houghton, A. Cruden, D. Infield, Modeling the benefits of vehicle-to-grid technology to a power system, IEEE Trans. Power Syst. 27 (2) (2012) 1012-1020. 